\title{
Non-invasive Optical End-to-End Test of a Large TMA Telescope (JWST) from the Intermediate Focus
}

Tony Whitman, ITT Scott Knight \& Paul Lightsey, Ball Mark Waldman, Sigma Space SPIE 7436-14 August 3, 2009 
- Traditional method for optical testing of telescopes

- JWST challenges

- JWST system - level optical test objectives

- Description of optical tests

- Wavefront sensing and test sources 
- Start with a point source, image pattern, or interferometer at the image plane of the telescope

- The light propagates through the telescope to form a collimated beam

- A flat test mirror reflects the collimated beam back to the telescope

- The telescope forms an image

- Image wavefront error = source wavefront error $+2 \times$ telescope wavefront error + test flat wavefront error

Flat Test

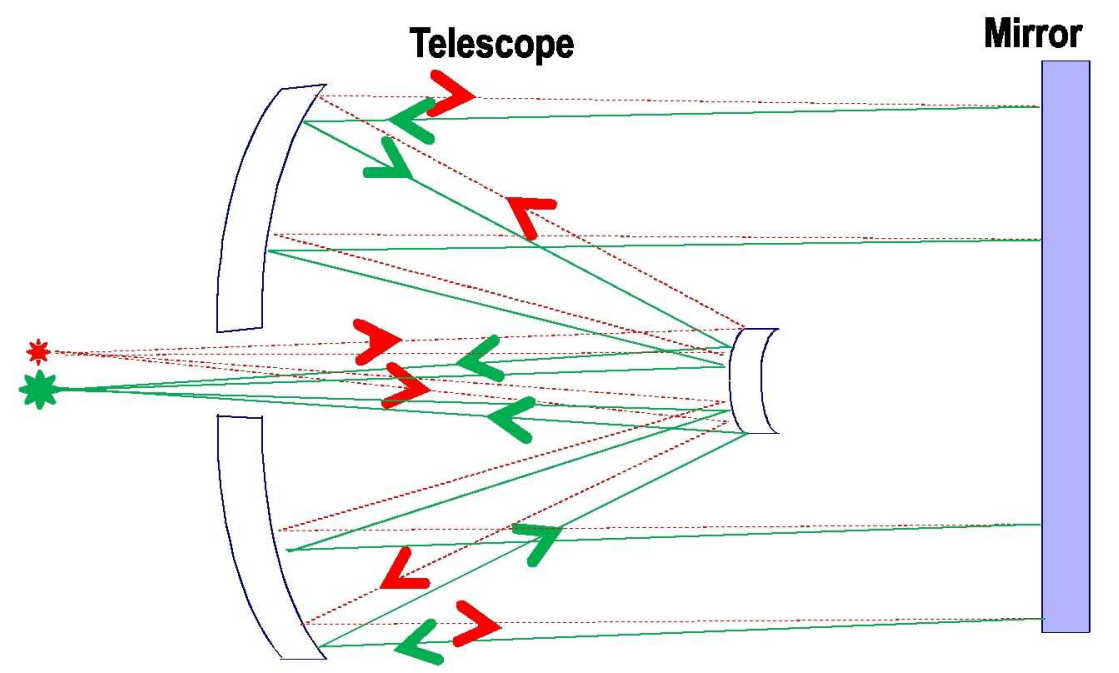


- JWST folds into the rocket, then redeploys and aligns on orbit

- Changes test from optical system performance to component performance and alignment capability

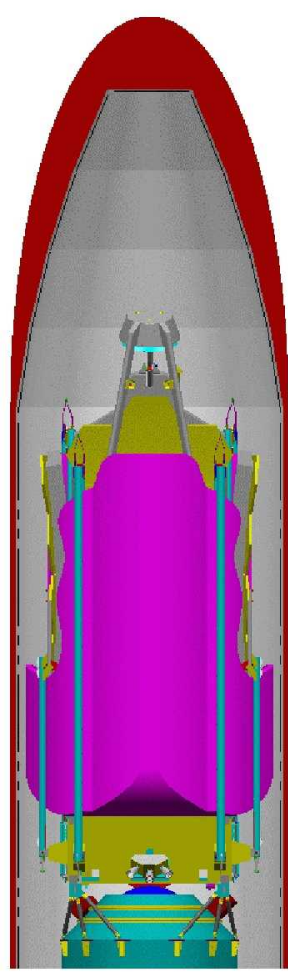

Stowed View

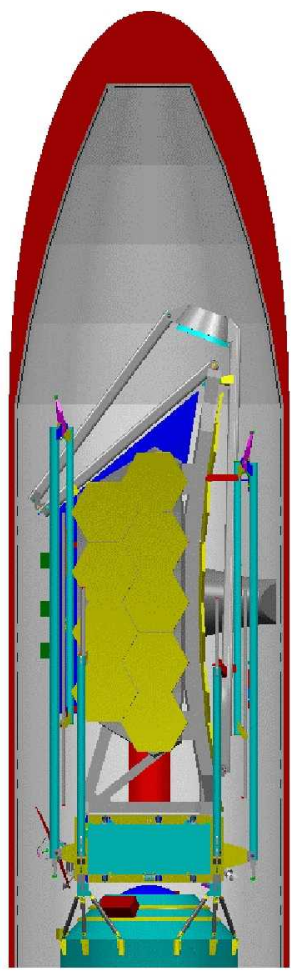

Without Sunshield

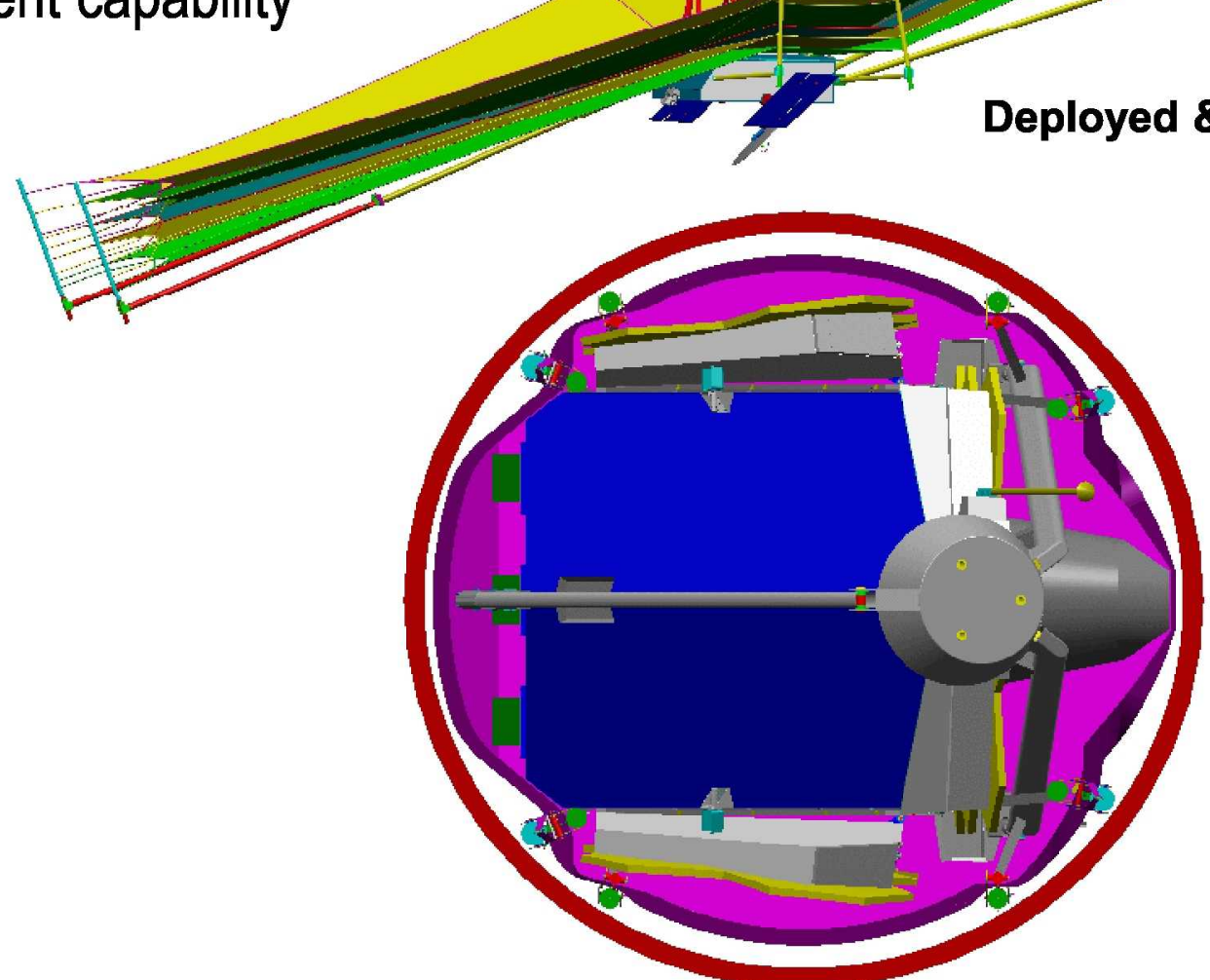

Top View of Observatory stowed in $5 \mathrm{~m}$ Ariane-5 Fairing 
- JWST folds into the rocket, then redeploys and aligns on orbit

- Changes test from optical system performance to component performance and alignment capability

\begin{tabular}{|ll|} 
& \multicolumn{1}{c}{ Alignable Optics } \\
ISIM & Deg. of freedom \\
\hline FGS_Guider & focus \\
FGS- Tuneable Filter & focus \\
NIRSpec & focus \\
NIRCam & tip/tilt, focus \\
& \\
OTE & \\
Primary Mirror segments & curvature, translation, rotation \\
Secondary Mirror & translation, rotation \\
Fine Steering Mirror & tip/tilt \\
\hline
\end{tabular}


- JWST folds into the rocket, then re-deploys and aligns on orbit

- Changes test from optical system performance to component performance and alignment capability

- Cost and stability of precision $6.6 \mathrm{~m}$ flat at cryogenic temperature to cover full aperture

- Smaller $1.5 \mathrm{~m}$ flat mirrors sample aperture

- No access to image surface in flight hardware

- Place test point sources at intermediate (Cassegrain) focus

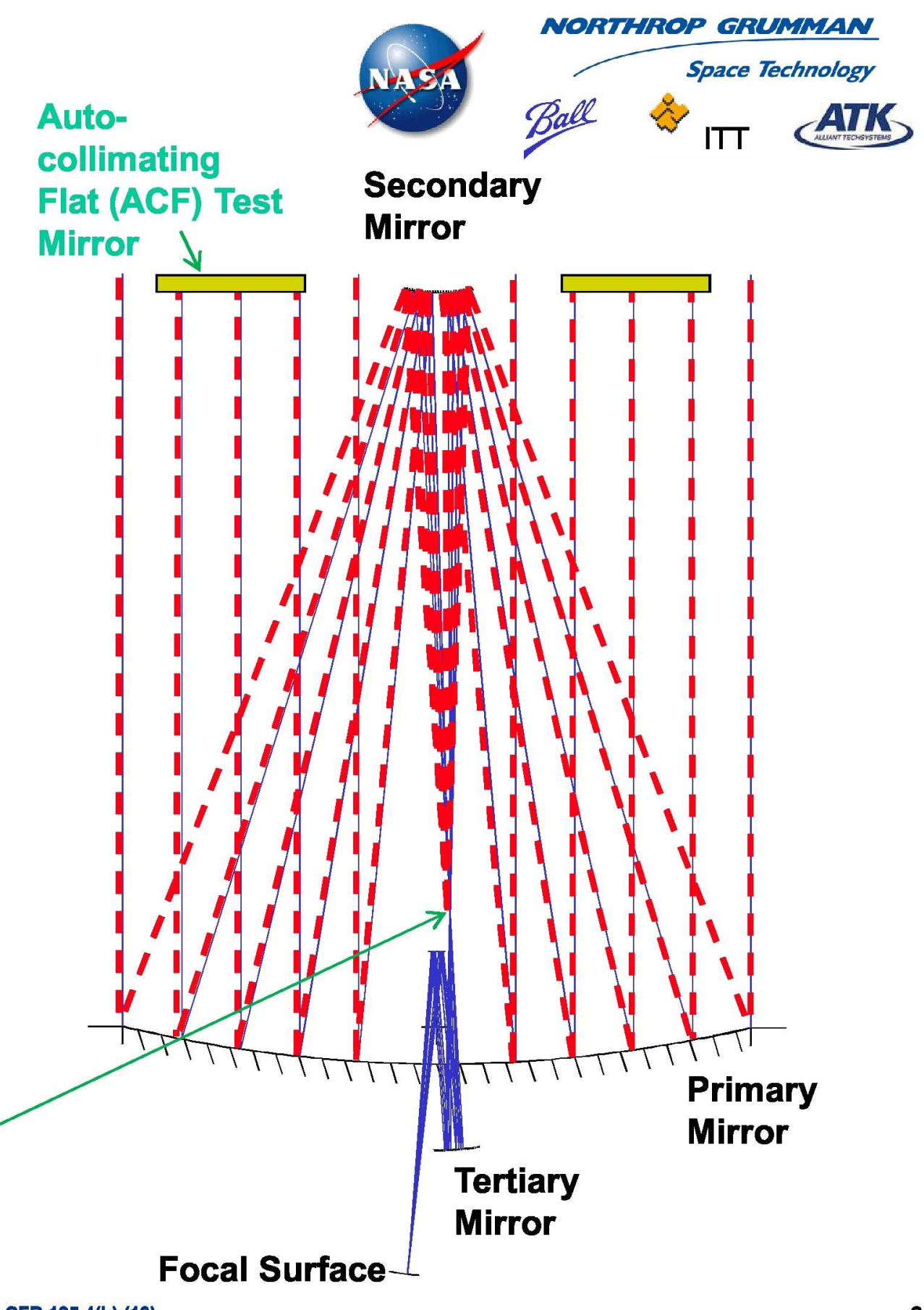


- JWST folds into the rocket, then re-deploys and aligns on orbit

- Changes test from optical system performance to component performance and alignment capability

- Cost and stability of precision $6.6 \mathrm{~m}$ flat at cryogenic temperature to cover full aperture

- Smaller $1.5 \mathrm{~m}$ flat mirrors sample aperture

- No access to image surface in flight hardware

- Place test point sources at intermediate (Cassegrain) focus

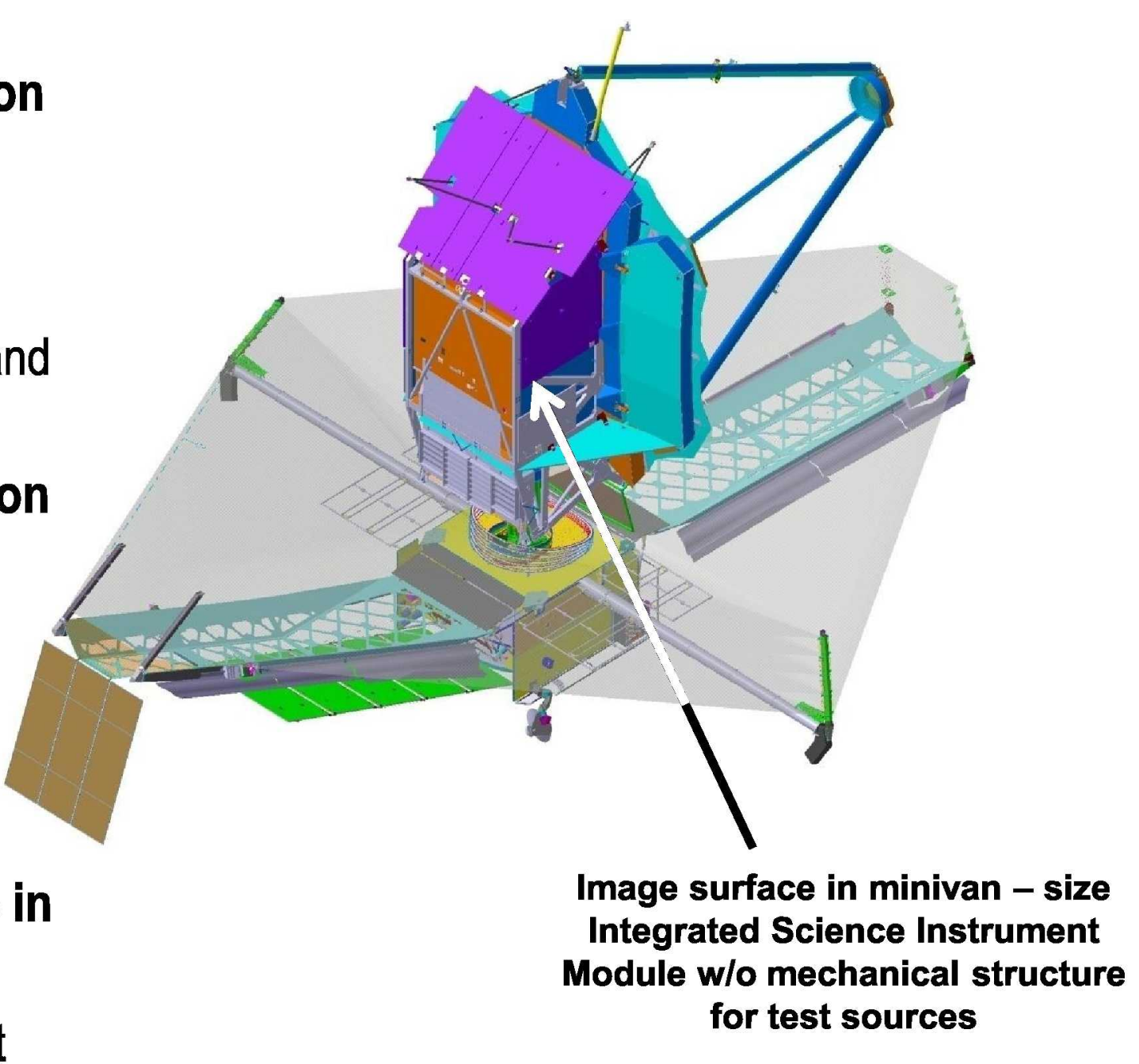


- JWST folds into the rocket, then re-deploys and aligns on orbit

- Changes test from optical system performance to component performance and alignment capability

- Cost and stability of precision $6.6 \mathrm{~m}$ flat at cryogenic temperature to cover full aperture

- Smaller $1.5 \mathrm{~m}$ flat mirrors sample aperture

- No access to image surface in flight hardware

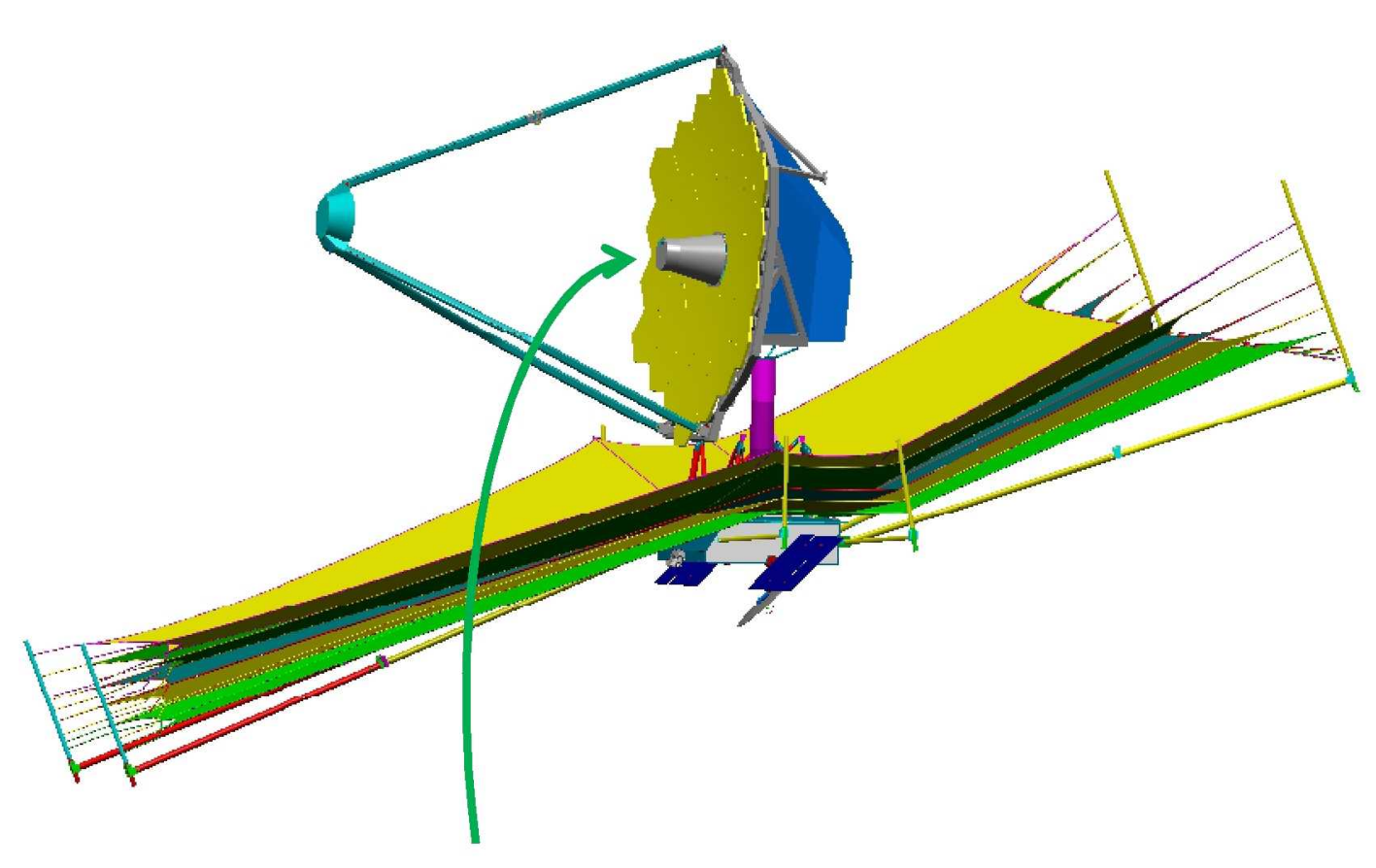

- Place test point sources at intermediate (Cassegrain) focus

\section{Cassegrain focus accessible outside Aft \\ Optics Subsystem (AOS) baffle}

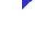




\section{Secondary}

Inward (downward) sources Mirror

illuminate Tertiary Mirror and

Science Instruments (blue lines)

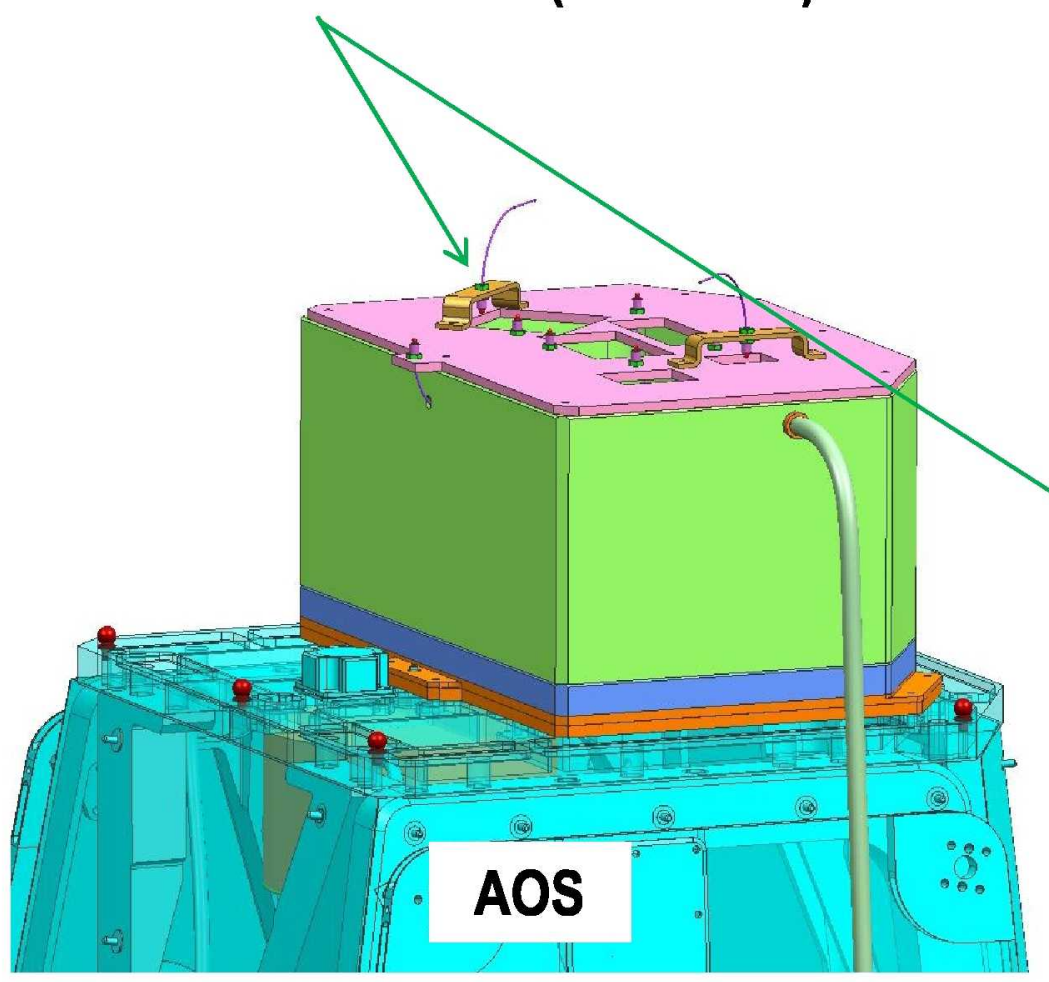

Focal Surface -
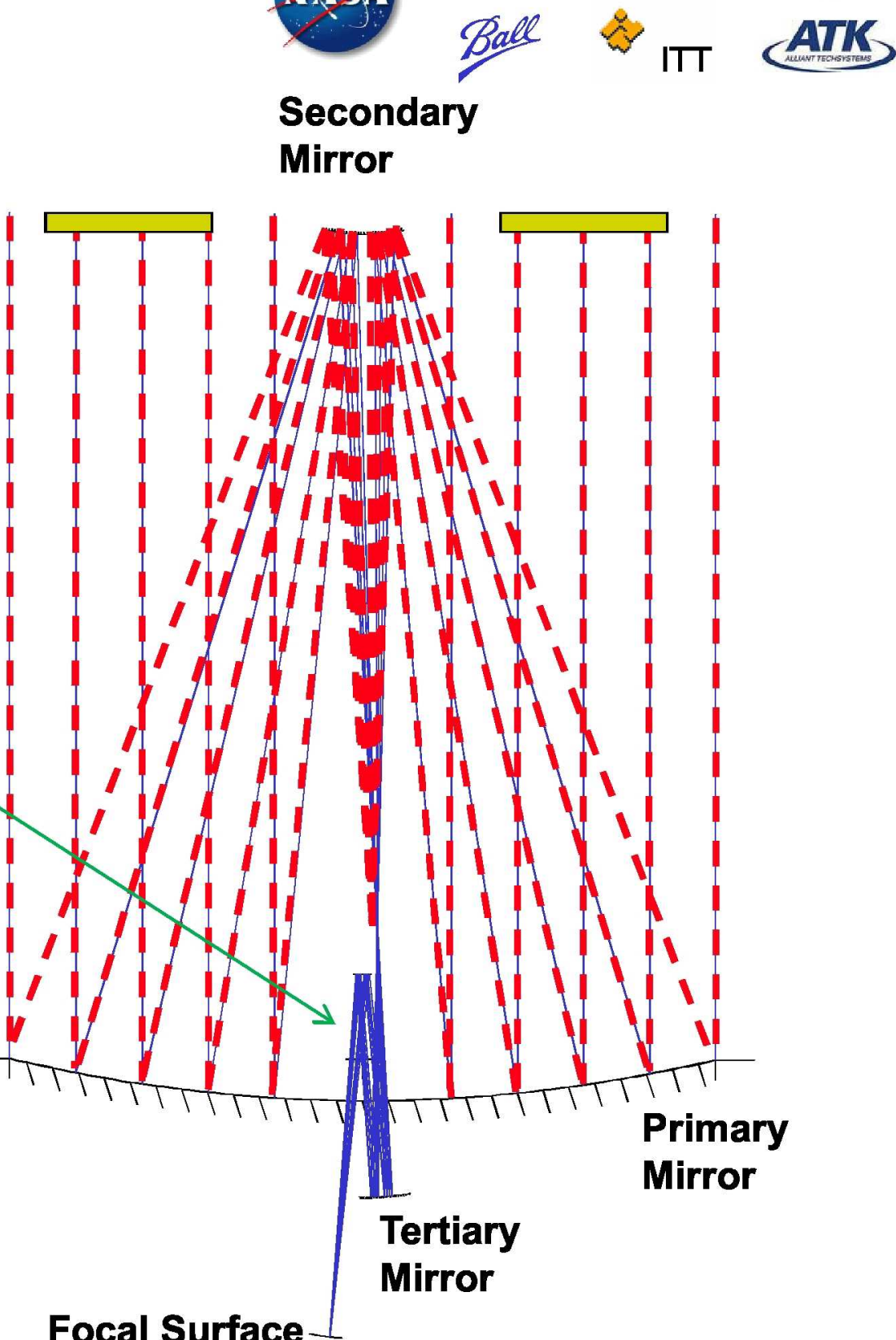
Outward (upward) sources illuminate Secondary Mirror, PM Mirror and ACFs before reflecting through whole system (red lines)

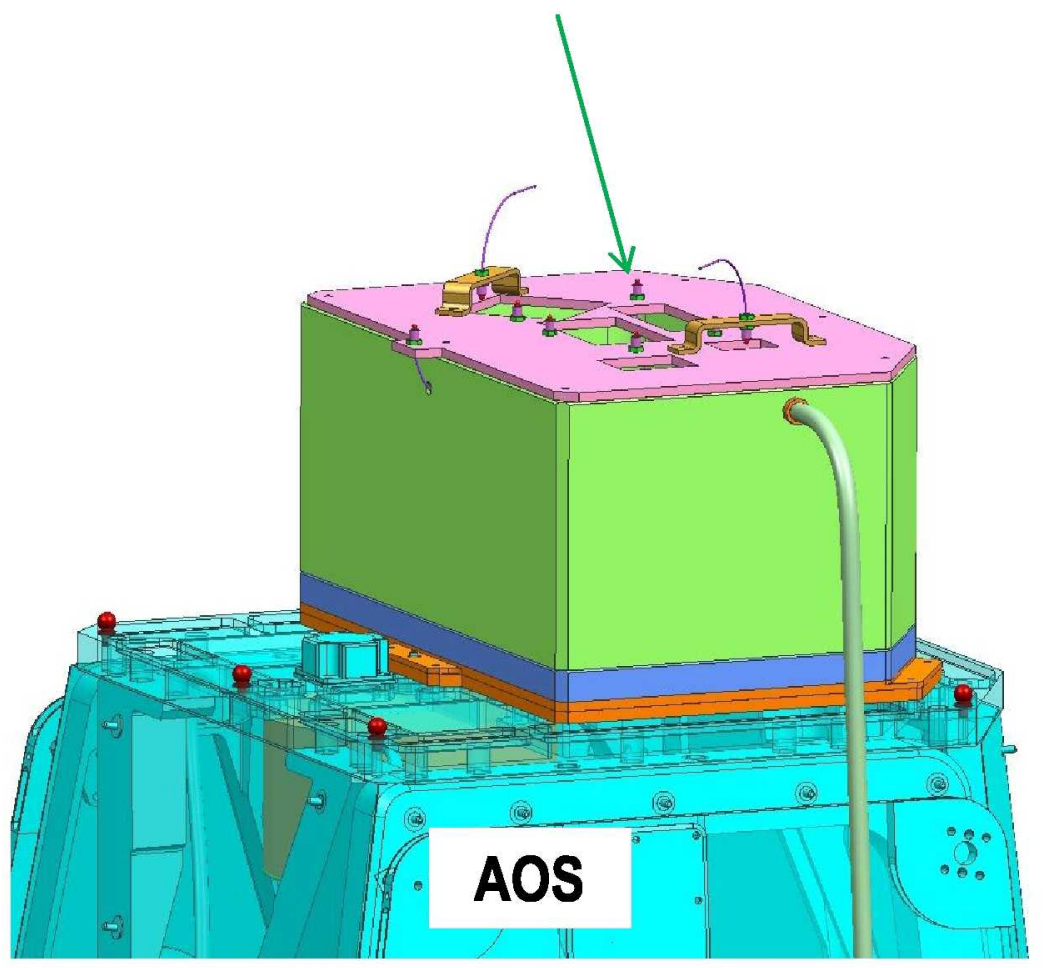

- "Pass and a half test" replaces "double pass" test

- WFE calculated from images captured by Science Instrument detectors using phase diversity

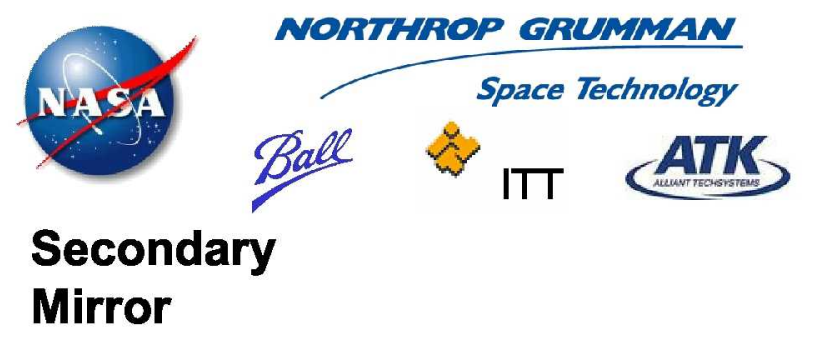

(1)
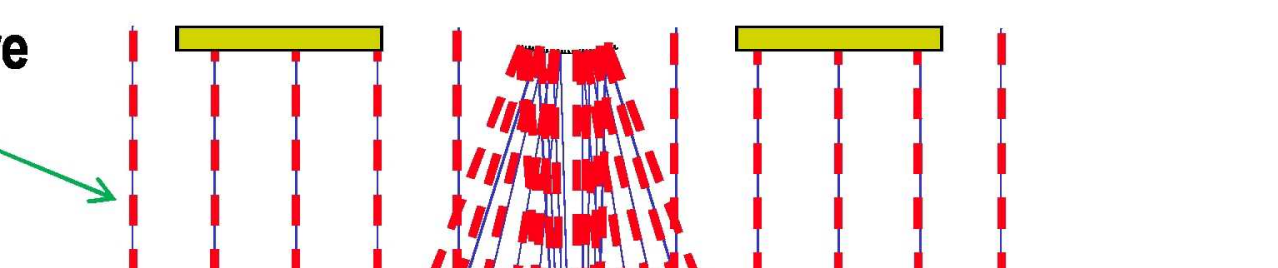


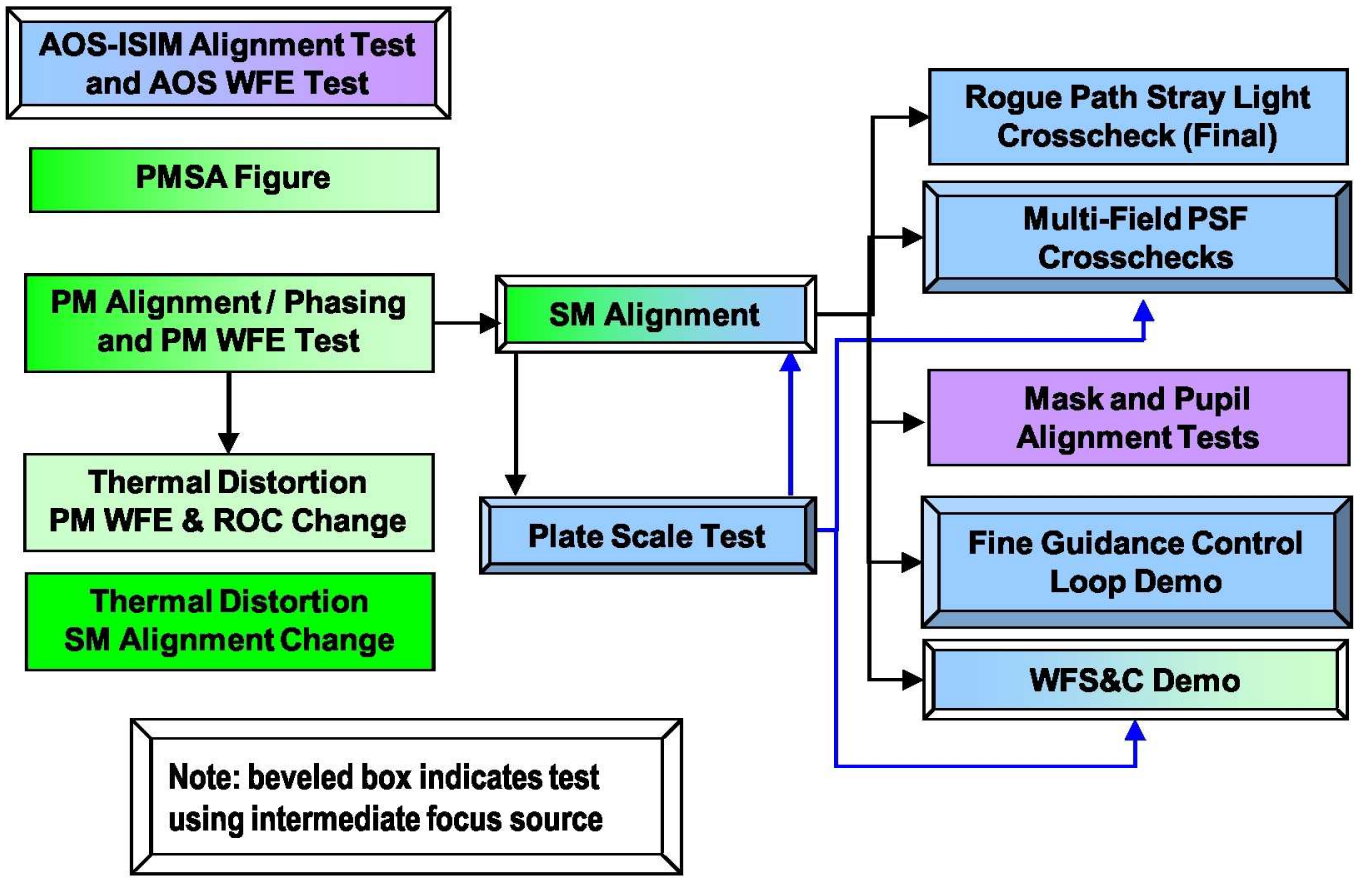

- AOS-ISIM Alignment Test

$>$ Inward sources test fixed AOS to ISIM alignment

- AOS WFE Test

$>$ WFE of inward source image with instrument WFE and source WFE removed = AOS WFE
- SM Alignment

$>$ WFE from outward source image through sampled aperture tests SM alignment to PM

- Plate Scale Test

$>$ Compare outward source image motion on detector with measured tilt of flat test mirror 


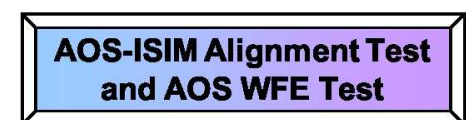

and AOS WFE Test

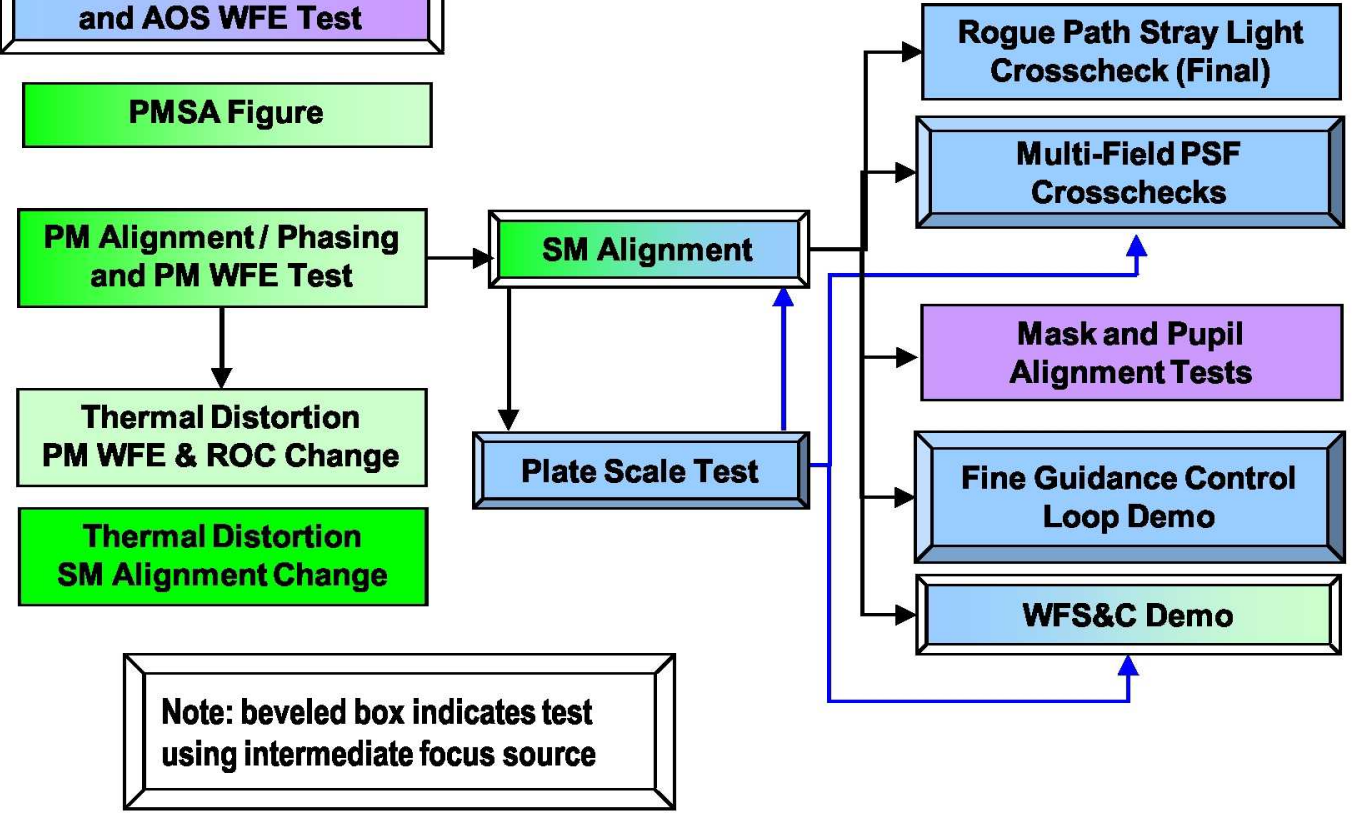

- Fine Guidance Control Loop Demonstration

$>$ Full system functional demonstration with inward and outward sources

- Wavefront Sense and Control Demonstration

$>$ Demonstration of on-orbit alignment methods
- Multi-Field PSF Crosschecks

Outward source image captured in every instrument for full optical system check (Tilting ACFs moves field location of images.)

$>$ Tilt of telescope focal surface relative to common instrument focal surface using images across field by outward sources 


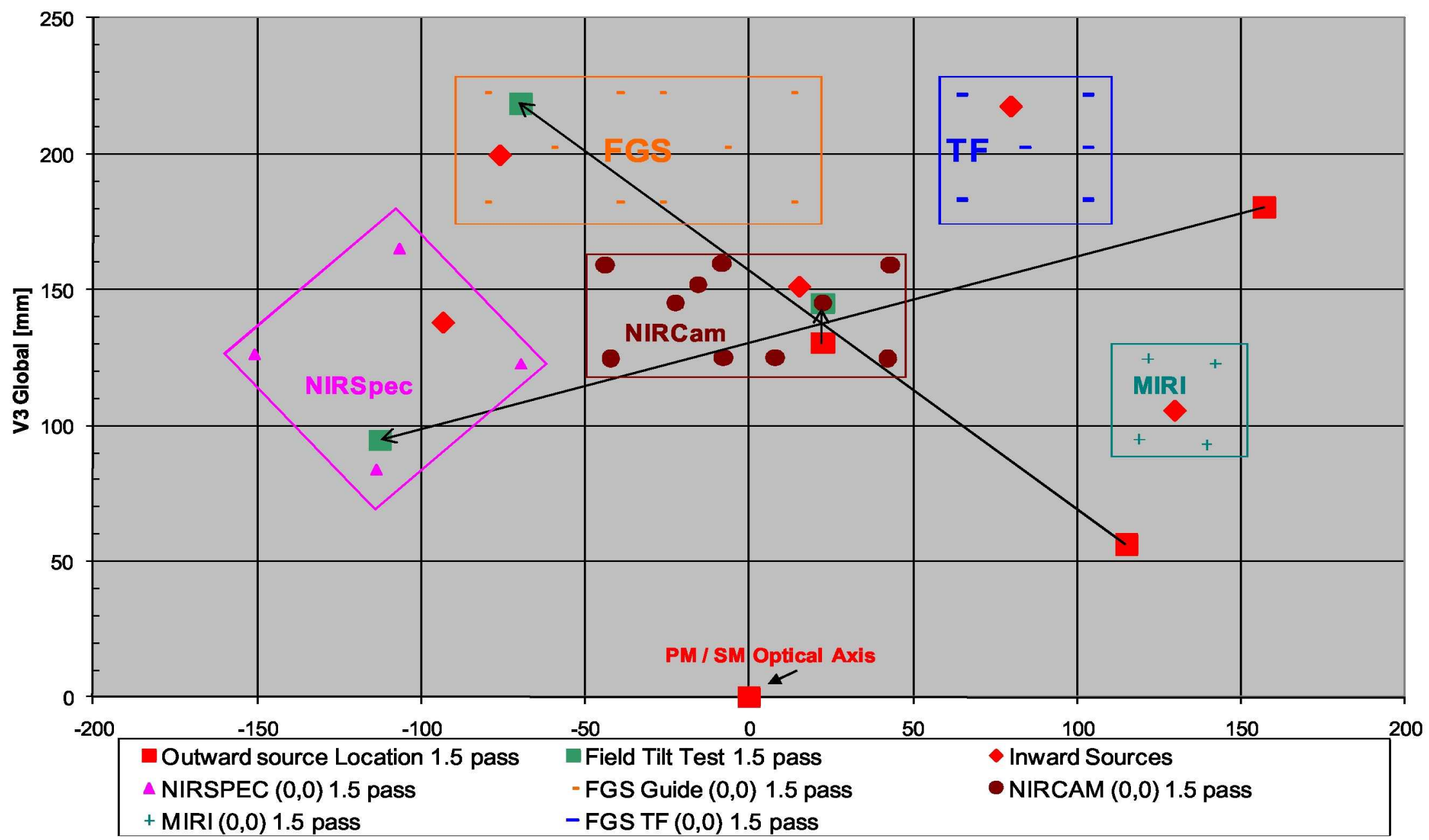


- Outward source on PM / SM optical axis has no significant additional WFE

- An off-axis anastigmatic telescope has WFE off-axis by design

- Tertiary Mirror designed to compensate for PM / SM field aberrations, particularly coma and astigmatism

- The phase retrieval algorithms developed for on-orbit alignment of JWST have sufficient dynamic range and sufficient target WFE range to extract system WFE while accommodating the large deterministic off-axis aberrations

- A centroiding algorithm for clocking and decenter measurments can also accommodate the large off-axis aberrations

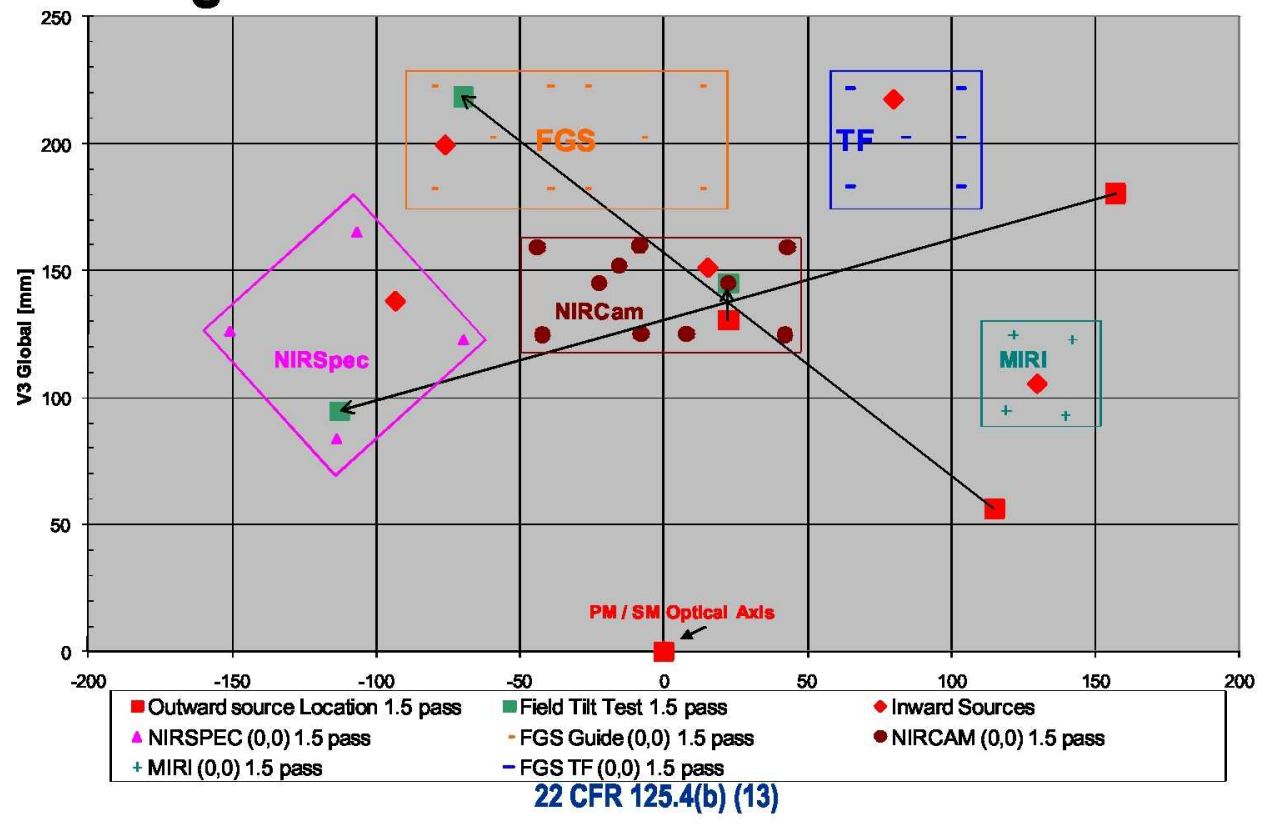




\title{
Non-invasive Optical End-to-End Test of a Large TMA Telescope (JWST) from the Intermediate Focus
}

\author{
Tony Whitman ${ }^{\mathrm{a}}$, J. Scott Knight ${ }^{\mathrm{b}}$, Mark Waldman ${ }^{\mathrm{c}}$, Paul Lightsey ${ }^{\mathrm{b}}$ \\ ${ }^{\mathrm{a}}$ ITT; ${ }^{\mathrm{b}}$ Ball Aerospace \& Technology Corp.; 'Sigma Space Corp.
}

\begin{abstract}
The James Webb Space Telescope (JWST) requires testing of the full optical system in a cryogenic vacuum environment before launch. Challenges with the telescope architecture and the test environment lead to placing removable optical test sources at the Cassegrain intermediate focus of the Telescope. The Science Instrument suite will be used to align the telescope and to verify the wavefront error. The Science Instruments capture test images that are analyzed using focus diverse phase retrieval. The wavefront sensing algorithms have the large dynamic range required to measure the relatively small wavefronts of interest in the presence of the large aberrations resulting from the off-axis source locations at the intermediate focus. These inherent aberrations of the off-axis design are removed analytically from the measured data. The test design and in-situ wavefront sensing process enables a number of tests to verify the alignment and optical quality of the system.
\end{abstract}

Keywords: JWST, telescope, test, optical source, intermediate focus, wavefront sensing

\section{INTRODUCTION}

Typically, space telescopes are optically tested as a system by placing a pattern of test sources or an interferometer source at the image surface that project through the telescope backwards to create a collimated beam exiting from the primary mirror. A flat test mirror reflects the collimated beam back through the telescope to create an image or interferogram (fringe pattern) of the test source(s) at the image surface. In other words, the telescope generates its own artificial star for a star image test. The images are then evaluated optically to verify the performance of the telescope before launching into orbit.

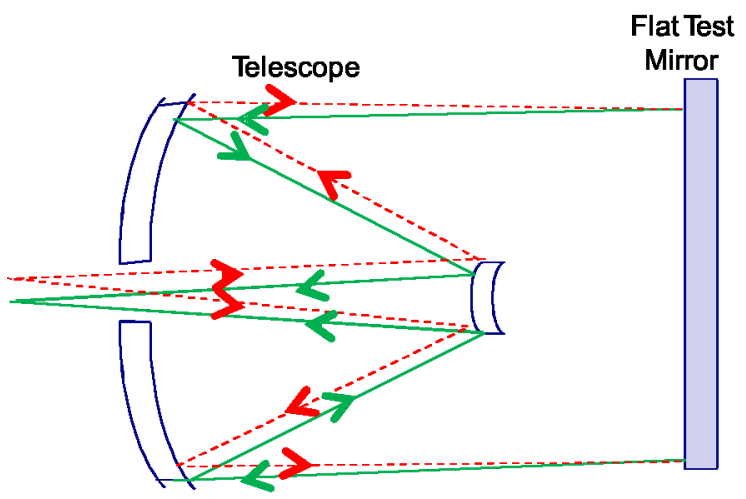

Figure 1 Schematic of test beam passing twice through a telescope under test using a flat test mirror to reflect the beam collimated by the telescope

JWST presents several changes to this approach [1]. Foremost, this telescope is folded after the ground testing into the launch configuration. Then the telescope redeploys and aligns on orbit. The ground optical test, therefore, must test the optical performance in one aligned state and also test the capability to re-align on orbit. The test breaks down into three categories: (1) tests of the surface figures of the optical components, (2) tests of the actuator positions for the adjustable components for a ground aligned telescope, and (3) tests of the alignments of the non-adjustable components of the telescope. ( 
Table 1 lists the operationally adjustable degrees of freedom.) The data from these three categories are used in analytic simulations of the on-orbit optical system and wave front sensing and control processes to verify that the optical performance during flight will meet requirements.

Secondly, for the traditional test method of reflecting the full aperture of the collimated beam, the sheer size of this telescope would require a large $6.6 \mathrm{~m}$ diameter test flat that would sag considerably from its own weight and offer significant stability challenges, particularly when cooling down to the cryogenic test temperatures necessary for JWST. The new approach uses smaller $1.5 \mathrm{~m}$ diameter flat mirrors to reflect enough of the collimated beam aperture to check the ground alignment and other functions of the full optical system.

Finally, the JWST design architecture results in limited access for test sources at the image surface of the telescope (also the object surface of the science instruments). The location is unreachable amidst large flight hardware in the surrounding area and lacks convenient support structure for test sources. On the other hand, an intermediate focal surface between the secondary mirror and the tertiary mirror is fully accessible and support structure can be mounted to the nearby flight hardware, the Aft Optical System (AOS), housing the Tertiary mirror and Fine Steering mirror (Figure 2). The AOS includes an entrance aperture located in close proximity to the intermediate focal surface created by the Cassegrain fore optics consisting of the segmented Primary mirror and the Secondary Mirror.

Although the intermediate focal surface is aberrated by the off-axis optical design (the field aberrations of the Cassegrain that are compensated by the off-axis tertiary mirror in normal imaging ), the test objectives can still be met by placing test sources at this surface. Below are a brief description of the planned tests with the role of the test sources, and a description of the methods for accommodating the source aberrations.

\section{DESCRIPTION OF TESTS}

\subsection{Test Overview}

Figure 2 illustrates the components of the JWST Integrated Science Instrument Module (ISIM) and Optical Telescope Element (OTE). The instruments are tested by the individual Science Instrument teams for optical performance before integration to the ISIM. After integration of all the instruments, the ISIM is tested optically and cryogenically with an OTE simulator (OSIM). The OSIM-ISIM test sets the focus and tilt adjustments of the instruments to align them to a common focal surface and to a common pupil for mating to the OTE, and checks the optical performance of the instruments after the common alignment. 


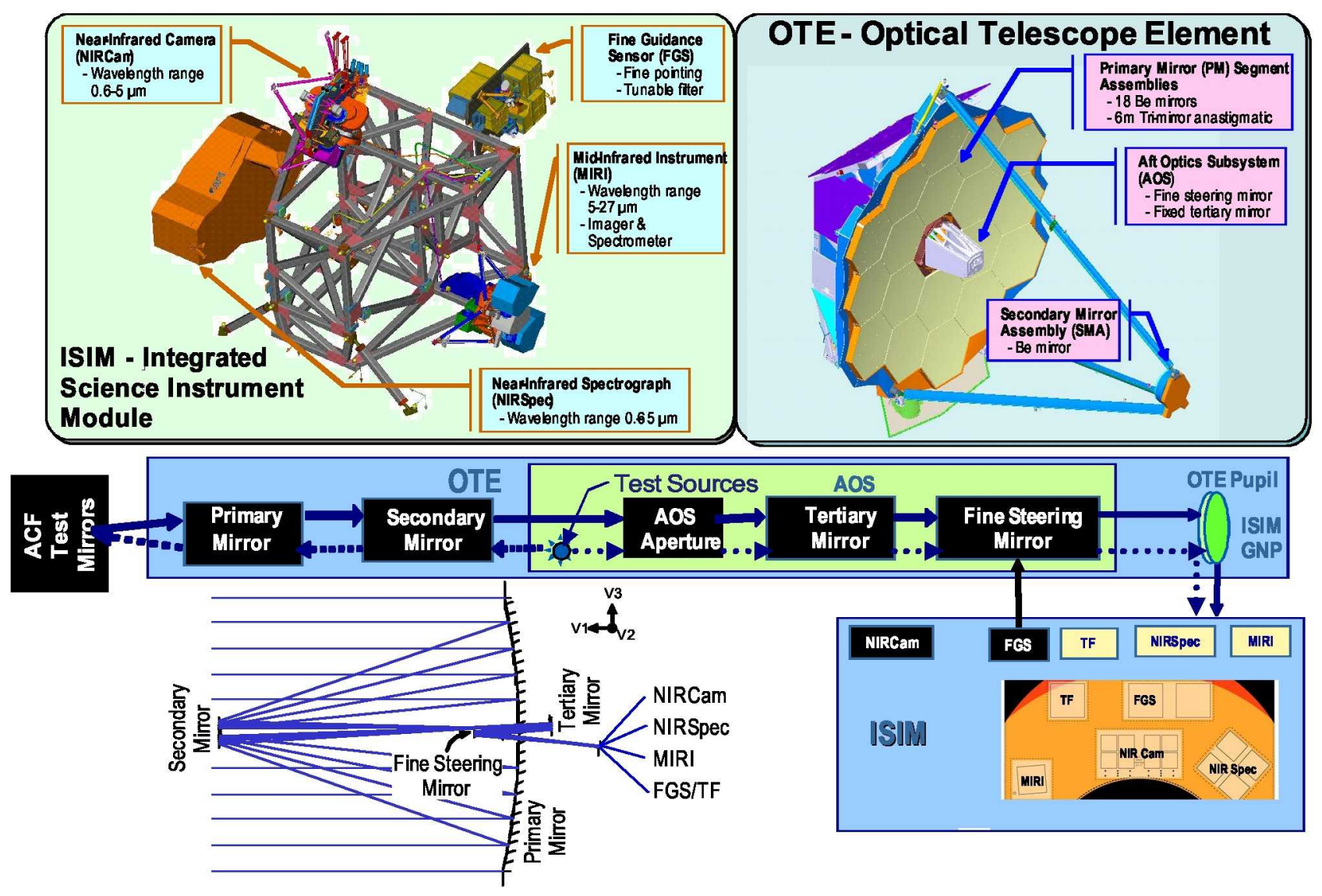

Figure 2. Telescope and instrument hardware and optical test block diagram.

Likewise, the Primary Mirror (PM) segments and Secondary Mirror (SM) are optically tested separately and cryogenically before integration to the OTE. The Fine Steering Mirror and Tertiary Mirror are optically tested separately before integration to the AOS. Then the alignment of the FSM and TM to AOS interface references is tested as a subsystem before integration to the OTE.

The OTE and ISIM are then integrated. The integrations at all levels are checked using optical metrology instruments (e.g. laser trackers) with the hardware at room temperature.

Therefore, the critical needs of the system level cryogenic optical test reduce to a check of the integrated segments to form a PM, a check of the cryogenic alignment of the ISIM to AOS, and the alignment of the SM/PM combination to the AOS, a check of any performance effects from the final integration steps, and a check of the optical sensitivity to temperature. Figure 3 lists all the key optical tests. The test sources at the intermediate focus are required for the tests in the beveled boxes in the figure.

Table 1. List of actuated degrees of freedom 


\begin{tabular}{|lr|}
\hline & Adjustable Optics \\
ISIM & Deg. of freedom \\
\hline FGS_Guider & focus \\
FGS- Tuneable Filter & focus \\
NIRSpec & focus \\
NIRCam & rotation, focus \\
OTE & \\
\hline Primary Mirror segments & curvature, translation, rotation \\
Secondary Mirror & translation, rotation \\
Fine Steering Mirror & rotation \\
\hline
\end{tabular}

Regarding the other tests in the figure, the integrated PM is tested using an interferometer at the center of curvature of the PM without the use of test sources within the telescope. The alignment of the SM and PM to the AOS is measured using photogrammetry methods with its own illumination sources and cameras in addition to the measurement in section 2.4. The entrance pupil of the telescope is the PM and is illuminated from sources outside of the telescope for registration of the PM for its alignment to other pupils in the system. The system sensitivity to temperature is dominated by changes in the shape of the PM and the alignment of the SM; both effects are tested by photogrammetry and metrology at the center of curvature of the PM.

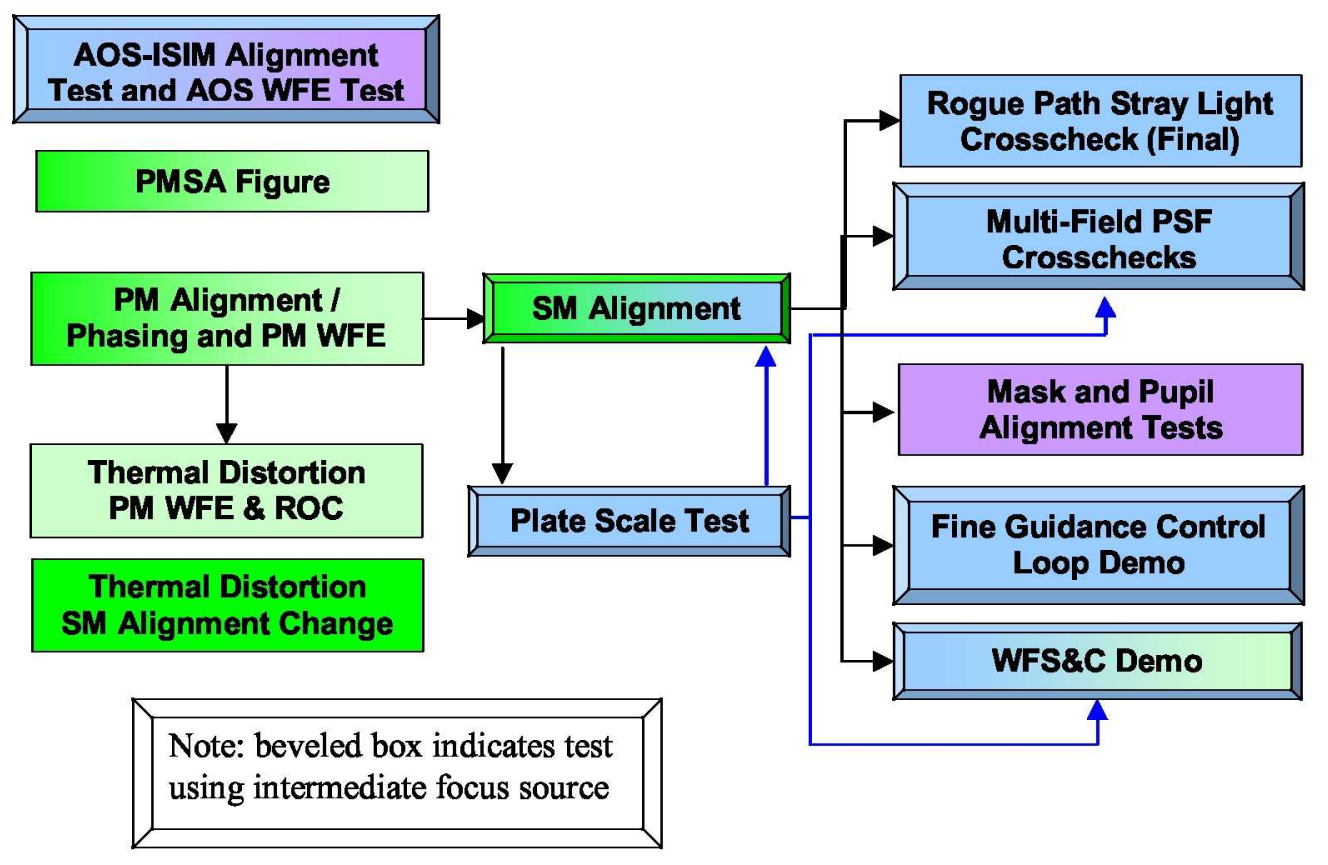

Figure 3. List of cryogenic tests and flow

\subsection{AOS-ISIM Alignment Test - alignment of the non-adjustable optics}

Optical test point sources aimed to emit inward towards the Tertiary Mirror are used to check the alignment of the AOS to the ISIM. The light in this case follows along the dotted arrow path in Figure 2. 
At the AOS-level, prior to integration to the OTE, the test sources are imaged by the AOS optics in a cryogenic test where the image locations are calibrated relative to the AOS optics. Then, at the system level, the image locations are captured by the pixel locations on the Science Instrument detectors. The pixel locations are compared to the image locations from the AOS-level subsystem test to determine the ISIM alignment relative to the AOS optics in lateral position as well as the clocking angle (roll). Since the images are highly comatic and astigmatic, extra analysis determines the centroid of the image as described in section 3.4. Wavefront sensing methods, described in section 3.3, are used to determine the amount of de-focus error in the images to determine average focus alignment between the AOS and the ISIM.

Although tilt (pitch and yaw) can be checked by measuring the change in focus across the field, tilt is more accurately determined by testing the shear alignment between the AOS pupil mask and the pupil of the NIRCam, one of the Science Instruments. The NIRCam has the capability to image the AOS pupil mask onto the detector by remotely inserting a lens system into the optical path. The mask is lit by one of the inward test sources on the AOS. The NIRCam also images lit references within the NIRCam onto the detector that indicates the NIRCam pupil position. The centroid of the NIRCam references on the detector is compared with the centroid of the pupil mask image to determine the relative shear between the pupils. The shear and the optical path length between the NIRCam pupil and the OTE pupil are used to calculate the relative tilt. The optical path length is calculated based on integration data and as-built design models.

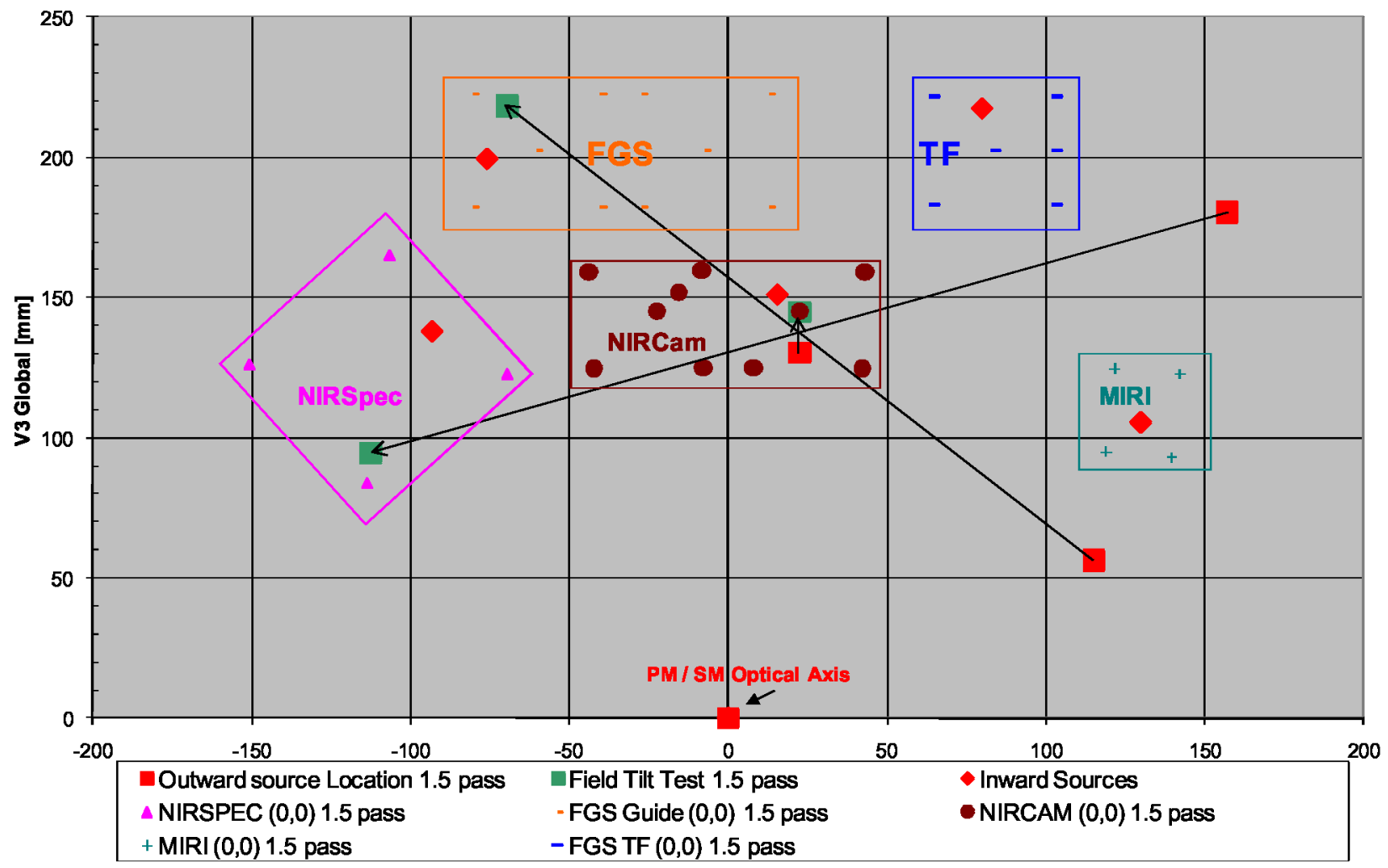

Figure 4 Test source image positions relative to instrument fields of view at the intermediate focus

\subsection{AOS WFE Test}

The AOS wavefront error (WFE) is tested as a subsystem prior to integration to the OTE. The AOS WFE Test checks the WFE after integration. The WFE of the Science Instruments are tested after integration to the ISIM with the OSIM. The inward source images are evaluated for WFE using the phase retrieval methods in section Error! Reference source not found. Then the WFEs for the instruments from the OSIM test are subtracted to yield the AOS WFE.

\section{CFR 125.4(b) (13)}




\subsection{Secondary Mirror (SM) Alignment - ground alignment of SM to PM}

Misalignment between the SM and PM results in aberrations of power, coma, and astigmatism. These aberrations in the full aperture manifest as tilt, power, and astigmatism in the sampling of the full aperture by the smaller test mirrors. The uncertainty in tilt of the test mirrors is too large for alignment determination, so only power and astigmatism is used in the sampled aperture images.

The test mirrors are pointed to aim images of the outward on-axis source into the NIRCam instrument. The images from each sampled aperture test mirror are separated to avoid the complicated image interference that would be produced by the unphased images. These images are evaluated for WFE in power and astigmatism, using phase retrieval, for each test mirror aperture. Optimizing the average power among the test apertures sets the SM axial position. Optimizing the power and astigmatism of the individual apertures optimizes the centering and tilt of the SM relative to the Primary Mirror (PM). (Rotational symmetry eliminates the need to measure clocking angle.)

In this case the outward source is nominally on the SM and PM optical axis. The WFE on the SM/PM axis is smaller than the off-axis sources by two orders of magnitude, allowing for increased test accuracy. However, due to the gravity induced wavefront error, the wavefont is still large compared to the desired on orbit wavefont. .

\subsection{Plate Scale Test}

This test is a calibration of the image translation on the detector relative to the angular change of the collimated beam entering the telescope. The on-axis $(0,0)$ source is used, so source aberrations are insignificant. Therefore, centroiding is simpler. A test mirror is tilted with a measured angle change while the image is tracked across the detector -specifically a NIRCam detector - to generate the plate scale. The plate scale along with the measured Primary Mirror diameter is used to calculate the effective focal length of the optical system. The OTE portion is estimated by extracting the previously measured focal length of the Science Instrument.

Plate scale is slightly sensitive to the SM axial position. However, this quality needs to be known accurately for the wavefront sensing method to determine SM alignment. Hence, special algorithms using the measured optical MTF cutoff cross-check and refine the measured plate scale information sufficiently for the phase retrieval algorithm.

\subsection{Multi-field PSF Cross Checks}

The on-axis $(0,0)$ source is imaged into many points in each instrument as a check on the full optical system using phase retrieval. The images are moved to the different field points by tilting the auto-collimating test mirrors.

An alternate configuration is used with multiple off-axis sources creating multiple images with the test mirrors in one ACF test mirror tilt position. This configuration gives the opportunity to capture multiple images in several instruments simultaneously. In particular, this configuration is used to measure the tilt of the telescope focal surface relative to the ISIM common object surface.

This alternate configuration is illustrated in Figure 4. The test mirror is aimed at the center of the " $\mathrm{X}$ " in the figure. In this position, 3 outward test sources depicted by squares in the figure on the lower or right end of the lines will reflect off the test mirror at equal and opposite field angles to image at the locations depicted by squares on the other ends of the lines. The difference in focus between the images gives the tilt in the focal surface.

In addition, the field tilt can be measured by comparing the change in focus from the image of the on-axis source as a test mirror moves the image to various points of the science instruments. This gives the advantage of a smaller aberration source and more than 3 points are possible. However, this method is not simultaneous and has the risk of additional uncertainty due to stability effects.

\subsection{Fine Guidance Control Loop Demonstration}

The Fine Guidance Control Loop includes the Fine Guidance Sensor (FGS) in the ISIM and the Fine Steering Mirror in the AOS. The mirror tilts in the opposite direction of the detected motion in the sensor to maintain guidance on the image position. They are performance tested individually before integration. At the system level, the loop is run mainly to check functionality and to check that correct polarity was maintained in the integration.

Figure 4 shows an inward test source for one FGS channel for a demonstration. The on-axis $(0,0)$ outward source can be reflected into both channels for a demonstration. In the reflected case, multiple test mirrors at different tilt positions can 
create multiple stars in the image to demonstrate identification of a target pattern. Furthermore, and a single image can be set in motion by tilting the test mirrors while operating the loop.

The normal centroiding algorithm for the loop is sufficiently capable of operating with the additional aberration content of the off-axis source and with the sampled aperture images of the test mirrors for this demonstration.

\subsection{Wavefront Sense and Control (WFSC) Demonstration}

WFSC is the process of moving the PM segments and the SM to align the telescope by optimizing the WFE of the light reaching the instruments. Similar to the FGC Loop demonstration, the performances of the WFSC components are tested individually before integration. The system level demonstration is mainly used to check functionality and integration workmanship since the gravity induced disturbance of the Observatory results in a different "best-aligned" condition than that expected for on-orbit operation. The on-axis $(0,0)$ outward test source is used to engage the SM and PM in the demonstration and to take advantage of the smaller aberration content for this source location.. The test mirrors are tilted to place the image in the field points needed for the demonstration. The normal algorithm is used in this demonstration with minor changes to parameters to accommodate the ground configuration (such as the additional optical path upon reflecting from the PM and SM twice and the smaller auto-collimating test mirror apertures). This demonstration covers nearly all aspects of the on-orbit process except the initial boresight determination and the phasing of all 18 primary segments. The ground system is incapable of providing an Observatory slew relative to a target star in the first case, and the sampled-aperture test mirrors only reflect light from 9 of the 18 primary mirror segments in the second case.

\section{WAVEFRONT SENSING AND CENTROIDING METHODS}

\subsection{Optical Configuration Implications for Wavefront Sensing}

The location of the off-axis test sources at the intermediate image of the three mirror anastigmat (TMA) telescope generates on the order of waves of coma and astigmatism. The desired wavefront sensing errors are close to lambda/ 100. The phase retrieval algorithms developed for on-orbit alignment [2] have a very large dynamic range. They are also capable of including large target wavefronts such that the difference between the target and the measured wavefront can be determined. Thus these algorithms are well suited to provide metrology for these tests.

\subsection{AOS Source Plate}

In normal JWST illumination, the tertiary mirror in the TMA corrects for the field error of the Primary and Secondary Mirrors. For the test locations in the inward direction, only the tertiary mirror of the TMA is illuminated and so it creates the very field dependant aberrations it is designed to correct. In the outward source illumination, a similar effect occurs since the light reflects off of the PM and Secondary mirrors twice but only once from the Tertiary mirror.

The sources are held in place by special ground test equipment, termed the AOS Source Plate, mounted onto the forward end of the AOS during ground testing. This removable plate holds the illumination devices in the correct design location for the intermediate focal surface and field location. The location and pointing direction of the sources are calibrated with respect to mechanical interface references on the AOS in a cryogenic vacuum test prior to integration to the OTE. The AOS with the plate of illumination sources become the master reference for the integration and alignment of the OTE. This is appropriate since the JWST architecture has the Tertiary Mirror and Fast Steering Mirror fixed relative to the stiff and stable AOS structure. The alignment of the ISIM with respect to the AOS is tested as described in section 2.2 .

An optical model developed from the as-tested AOS is used to predict the level of aberrations at the source field locations. This model is validated during a cryogenic AOS test series. The predictions are used to generate target maps for the system tests at the field locations. Then the wavefront difference between these design locations and the tested system are measured. The critical measurements use the focus wavefront. The field locations by themselves do not produce focus aberration, but focus aberration is produced by the position of the ISIM relative to the AOS (and AOS source plate) giving the desired alignment signature. Therefore, modeling errors do not affect these measurements. The AOS wavefront error test and SM alignment tests do include coma and astigmatism. Modeling errors for these tests are included in those test uncertainties. 


\subsection{Wavefront Sensing}

The phase retrieval algorithm uses defocused images to iteratively estimate the phase. The defocus is imparted to the system using the focus adjustment mechanism of the Science Instruments for the inward source test. The Mid Infrared Instrument (MIRI) does not have a means to adjust focus. So in this case, multiple sources are displaced from the ideal focal surface on the AOS Source Plate to provide defocus. For the outward test, the Secondary Mirror or Science Instrument focus adjustment mechanism can provide defocus. The Hybrid Diveristy Algorithm (HDA) [2] designed for on-orbit wavefront sensing is well-suited to the dynamic range needs for this test. Computer simulations including the science instrument detector performance have been performed in order to establish sensing uncertainties meeting the test objectives.

\subsection{Centroiding method}

In order to measure the translational and clocking alignment of the AOS to the ISIM, the image position and relative image positions are used. Due to the degree of non-symmetric aberrations in this test, simple center of mass centroids produce unacceptably large uncertainty. Instead, the method used is based on the property that the centroid of a diffracted point spread function is equal to the slope of the imaginary part of the diffraction optical transfer function at the origin [3]. This method was found to have adequately small uncertainty in the test environment. It compares well to the theoretical shift in centroid due to a comatic image [4].

\section{CONCLUSION}

Optical testing of the OTE / ISIM is accomplished with the use of light sources at the intermediate focus of the telescope. This solution meets test objectives while avoiding the access difficulty and interface complexity of placing sources at the image plane of the telescope as is normally done in traditional system optical tests. It allows the critical measurements of the Observatory to be made without a full aperture test mirror. The test program utilizes the in-situ Science Instruments themselves as the optical wavefront metrology, by using phase retrieval techniques. The off-axis optical design adds considerable aberration content to the test with the sources at this location. However, the deterministic content can be accommodated using the high dynamic range flight phase retrieval algorithms and modeled data

\section{ACKNOWLEDGEMENTS}

The JWST Test team was supported by the JWST contract NAS5-02200 with NASA GSFC. The JWST system is a collaborative effort involving NASA, ESA, CSA, the Astronomy community and numerous principal investigators. The test plan and analysis was accomplished through the efforts of individuals at ITT Space Systems, Ball Aerospace and Technologies Corp., and Northrop Grumman Aerospace Systems. The test plan and analysis has been vetted with Goddard Space Flight Center and several independent review teams.

\section{REFERENCES}

[1] C. Atkinson, J. Arenberg, G. Matthews, M. Waldman, A. Wertheimer, T. Whitman, and J. Oschmann, "Architecting a revised optical test approach for JWST" SPIE 7010-26 (2008)

[2] B. Dean, S. Acton et al, Phase Retrieval Algorithm for JWST Flight and Testbed Telescope”, Proceedings of SPIE, ,Vol. $6256(2006)$

[3] Virendra N. Mahajan, "Optical Imaging and Abbertions”, pg. 72, SPIE Press, (2001)

[4] Virendra N. Mahajan, “Abbertion Theory Made Simple”, Chapter 10, pg 131, SPIE Press,(1991) 